\title{
Axonal refractory period of single short toe extensor motor units in man
}

\author{
JÖRGEN BORG \\ From the Neurological Clinic, Karolinska Hospital, Stockholm, Sweden
}

SUMMARY The aim of this study was to test the reliability of the commonly used blocking technique for measuring the conduction velocity spectrum of the peripheral motor nerve in man, as first described by Hopf in $1962^{1}$. Electromyographic recordings were carried out with a selectivity permitting identification of single motor unit potentials. The conduction velocity of the single alpha motor axon was determined by a direct technique and by the blocking technique. The refractory period was determined by comparison of the results of these two techniques. The refractory period was studied in relation to the strength of the testing stimulus, axonal conduction velocity, skin temperature and age of the subject. When the strength of the test stimulus increased from $10 \%$ to $50 \%$ above the axonal threshold, the refractory period decreased from 1.7 to $0.6 \mathrm{~ms}$. An inverse relationship between the axonal conduction velocity and the refractory period was observed. When skin temperature was $4^{\circ} \mathrm{C}$ subnormal the refractory period was significantly prolonged; at $10^{\circ} \mathrm{C}$ subnormal it was more than doubled. The same relationship between axonal conduction velocity and the refractory period was observed in both young and elderly subjects. The significance of these findings for the clinical application of the blocking technique is discussed.

By electrical stimulation of a human motor nerve at two different points and electromyographic recordings, the conduction velocity of the fastest conducting alpha motor axons of the stimulated nerve can be calculated by dividing the length between the stimulus points by the latency difference of the electromyographic responses. ${ }^{23}$ This "direct" technique gives no detailed information of the conduction velocity of the small, slowly conducting motor nerve fibres.

Methods using blocking nerve impulses have been used for calculating conduction velocity of the slow nerve fibres. ${ }^{14}$ The "blocking" technique described by Hopf ${ }^{1}$ has been used in several clinical studies. ${ }^{\text {5-12 }}$ Two supramaximal stimuli are applied at a short time interval to a nerve. The first stimulus is applied close to the muscle evoking a maximum muscle response and also an antidrome nerve impulse volley. The second stimulus is applied at a greater distance from the muscle evoking a muscle response which, due to blocking by the antidromic impulse volley, alters in amplitude when the interstimulus time is

Address for reprint requests: Dr Jörgen Borg, Neurological Clinic, Karolinska Hospital, Fack, 10401 Stockholm, Sweden

Accepted 11 April 1980 changed. By determining the shortest interstimulus time for the maximum and the minimum second muscle response and correcting for the refractory period of the nerve fibres, the conduction velocity of the slowest and the fastest conducting fibres can be calculated.

Conflicts are found in the reported findings involving the use of this blocking technique. Hopf $^{1}$ first reported a difference of 4 to $7 \mathrm{~m} / \mathrm{s}$ between the conduction velocity of the fast and the slow motor fibres in the ulnar nerve, but after modifying the technique he described a difference of 6 to $10 \mathrm{~m} / \mathrm{s} .^{5}$ Blackstock et al ${ }^{11}$ found the difference in the same nerve to be 15 to $20 \mathrm{~m} / \mathrm{s}$. In the peroneal nerve Miglietta ${ }^{6}$ found a difference of 4 to $8 \mathrm{~m} / \mathrm{s}$. Betts et al ${ }^{12}$ (1976) stressed the importance of the correction made for the refractory period and regarded discrepancies in this respect as one important reason for the conflicting results.

The refractory periods of whole human peripheral nerve trunks have been studied extensively. ${ }^{12-18}$ The absolute and relative refractory periods of different kinds of single nerve fibres have been studied in animal experiments (see review by Paintal ${ }^{19}$ ). Due to methodological difficulties this has not been possible in man. 
Bergman $^{20}$ studied the recovery cycle of single motor axons in man by percutaneous recordings, but could study only part of the cycle.

In a previous study ${ }^{21}$ the axonal conduction velocity and discharge properties of single motor units in the short toe extensor in man were investigated using special muscle preparations (see Methods below). Such muscle preparations provided an opportunity of comparing the conduction velocity of the single alpha motor axon obtained by separate proximal and distal nerve stimulation, that is, by the direct technique, with the conduction velocity of the same motor axon obtained by the blocking technique. By comparing the results of these two techniques an index of the refractory period of the motor nerve fibre could be obtained. For the clinical application of the blocking technique, with regard to the variation in terms of stimulus strength, temperature and age of the subject, 71 motor neurons falling within the whole conduction velocity spectrum were studied.

\section{Methods}

The study included 12 clinically healthy subjects with normal motor conduction velocity (MCV), (the author, two students aged 25 and 30 years, and nine elderly subjects from a pensioners' gymnastic group aged 67 to 78 years).

The common peroneal nerve was stimulated close to the head of the fibula. The deep peroneal and the accessory deep peroneal nerves were stimulated at the ankle, anterior and posterior to the lateral malleoli. The stimuli were delivered through surface electrodes $0.6 \mathrm{~cm}$ in diameter. The cathode was placed over the nerve and the position was adjusted so that minimum stimulus strength was required for muscle response. The anode was placed $2-3 \mathrm{~cm}$ medial or lateral to the cathode. Rectangular pulse waves of $0.2 \mathrm{~ms}$ duration were used. Stimulus strength could be gradually changed between 0 and 300 volts.

Electromyographic recordings were made by bipolar needle electrodes (DISA 9013K0802 Electronic, Skovlunde, Denmark). Only motor units with potentials which could be safely identified by their characteristic shape in both voluntary contraction and at nerve stimulation were studied. The potentials were amplified and displayed on a Medelec oscilloscope no 4329, and recorded on Kodak Linograph direct print paper.

Selective electromyographic recordings from single motor units were obtained in three different ways. 23 motor units were studied in JB, who had taken part in earlier electromyographic studies of the same muscle resulting in an increased muscle fibre density within the motor unit and a decreased number of motor units permitting recordings from individual motor units. ${ }^{21}$ In the elderly subjects 45 motor units were studied. In this age group the normal typegrouping of the short toe extensor muscle ${ }^{22}$ is enough to permit recordings from individual motor units in some subjects. Three motor units were studied in two medical students who had an abberant innervation of the short toe extensor muscle by an accessory deep peroneal nerve ${ }^{23}$ passing posterior to the lateral malleol. When the main deep peroneal nerve was blocked by lidocain, only a few motor units were innervated by the accessory branch and selective recordings from these were possible.

After identification of a motor unit in a maximum sustained voluntary contraction, the effort was reduced until the motor unit no longer discharged. The direct technique for determining the conduction velocity was used first. The nerve was stimulated separately at the ankle and the fibular head, permitting the latency difference to be determined and the conduction velocity to be calculated. Stimulus strength was $10 \%$ above threshold for the test unit. The blocking technique was then applied to the same motor unit. The delay of the stimulus at the fibular head, usually $10-15 \mathrm{~ms}$, was initially long enough to permit the antidromic impulse from the ankle to pass the fibular head before stimulation there. The interstimulus time was then reduced by $0.2 \mathrm{~ms}$ stages until blocking took place at the fibular head due to refractory delay. The shortest interstimulus time without blocking occurring could then be determined. By comparing this time with the latency difference at separate proximal and distal stimulation, the refractory period of the test motor unit could be calculated. The stimulus strength at double stimulation was at first $10 \%$ above threshold. If there was no intolerable pain, the stimulus strength at the fibular head was made $25 \%, 50 \%$ and $100 \%$ above threshold.

Room temperature was held at $23-25^{\circ} \mathrm{C}$. Skin temperature at the fibular head was $30-32^{\circ} \mathrm{C}$. During the examination the temperature was regulated continuously by a Disaheater (DISA Electronic, Skovlunde, Denmark). The effect of low temperature on the refractory period was studied by cooling an area about $10 \times 10 \mathrm{~cm}$ around the fibular head using cold packs (ColPac).

\section{Results}

Figs 1 and 2 illustrate the axonal conduction velocity measurement in a motor unit by the direct technique and by the blocking technique.

Fig 1A shows the isolated motor units active on maximum voluntary effort. The motor unit with the highest amplitude potential was used is the test unit. Fig 1B shows the test unit at stimulation at the ankle and fig $1 \mathrm{C}, \mathrm{D}$ at stimulation at the fibular head. The stimulus strength at the fibular head was in $\mathrm{C} 10 \%$ above threshold and in $\mathrm{D} 100 \%$ above threshold. At the high stimulus level there was a contamination of the 

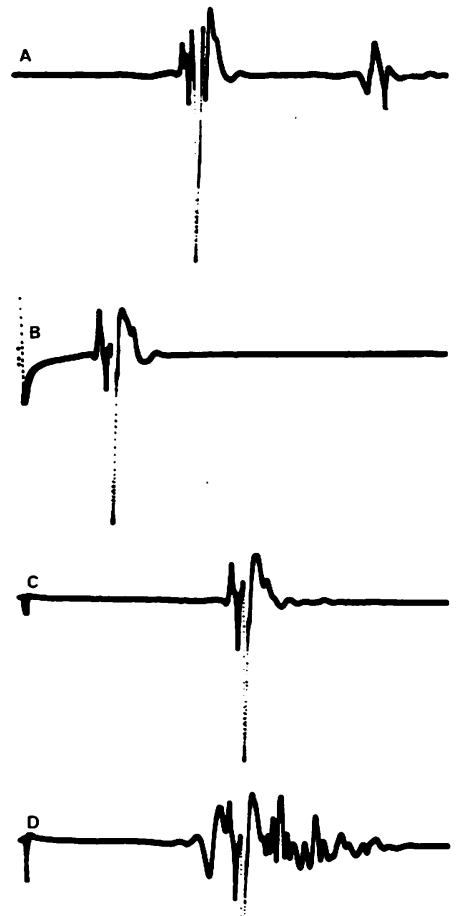

Fig 1 Axonal conduction velocity measured by direct technique. A: the motor units active in maximum voluntary effort. B: test unit at stimulation at the ankle. $C$ : test unit at stimulation at the fibu!a heat $10 \%$ above threshold. D: test unit at

stimulation at the fibula head $100 \%$ above threshold. Time bar $10 \mathrm{~ms}$.

test unit potential due to synchronisation of distant activity. The latency difference at the $10 \%$ level was $12.3 \mathrm{~ms}$, and it was the same at the $25 \%$ and $50 \%$ levels. At the $100 \%$ level it was reduced to $12.1 \mathrm{~ms}$ presumably due to stimulus spread (see below). The conduction velocity calculation was based upon the latency difference at the low stimulus level. The distance between the stimulus points was $39 \mathrm{~cm}$ and thus the axonal conduction velocity was $32 \mathrm{~m} / \mathrm{s}$.

As can be seen in fig 1 , a slight difference occurred in the shape of the action potential during a voluntary contraction compared with the same action potential evoked by electrical nerve stimulation. This was due to minor changes in the position of the needle electrode when muscle contraction was reduced from maximum voluntary effort to relaxation before nerve stimulation. Only a slight change of the shape was accepted and the motor unit identity was established by the absence of any other similar action potential on maximum voluntary effort, or at supra-maximum nerve stimulation, and also was confirmed by the blocking procedure (see below).

Fig 2 shows the results of blocking experiments using the same test unit as above. The stimulus strength at the fibular head was $10 \%$ above threshold in A-C, $25 \%$ above threshold in D-F, $50 \%$ in G-I and $100 \%$ in J-L. At each stimulus level the interstimulus time was reduced by 0.2 ms stages until blocking took place (C, F, I, L). The shortest time interval without blocking was $14.0 \mathrm{~ms}$ at the $10 \%$ level (B), $13.0 \mathrm{~ms}$ at the $25 \%$ level (E), $12.8 \mathrm{~ms}$ at the $50 \%$ level $(\mathrm{H})$ and
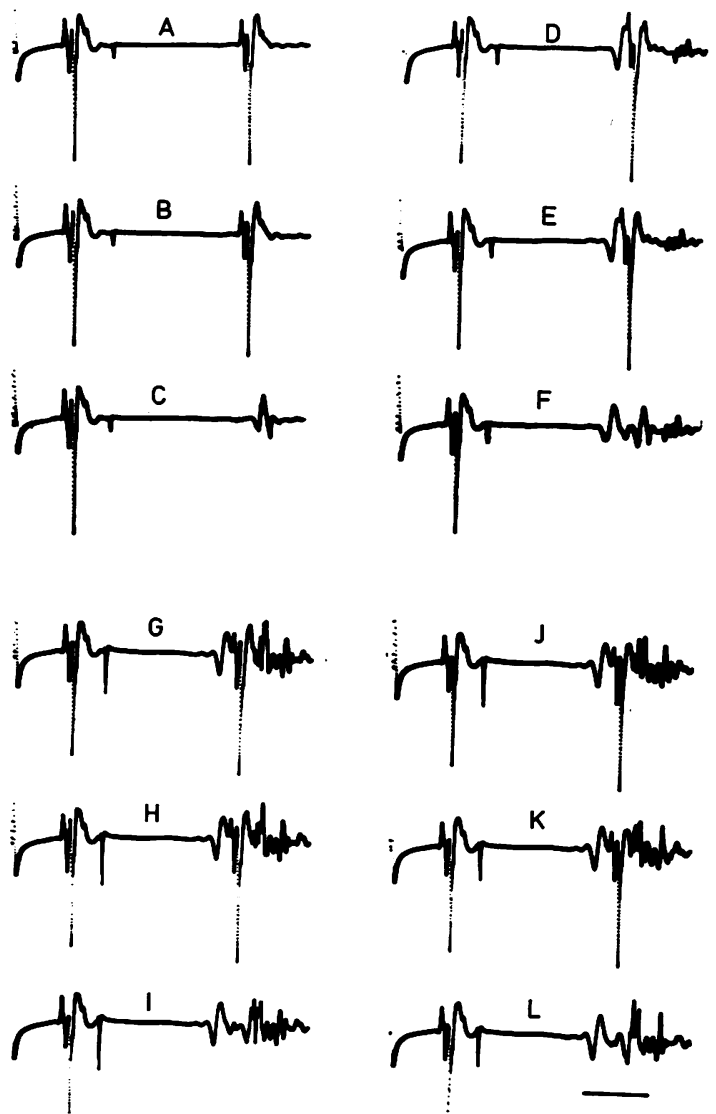

Fig 2 Axonal conduction velocity of the same test unit as in fig 1 measured by the blocking technique. $A-C$ : stimulus strength at the fibula head $10 \%$ above threshold, D-F: $25 \%$ above threshold, G-I: $50 \%$ above threshold, J-L: $100 \%$ above threshold. At each stimulus level the interstimulus time was reduced on $0.2 \mathrm{~ms}$ stages. Time bar $10 \mathrm{~ms}$. 
$12 \cdot 6 \mathrm{~ms}$ at the $100 \%$ level (K). With the direct techniques the latency difference was 12.3$12 \cdot 1 \mathrm{~ms}$. Thus, the shortest time interval without blocking was always longer than the latency difference of the direct techniques.

Since the axonal conduction velocity is regarded as being the same in the antidromic and orthodromic directions of a nerve fibre and since the measurements were made under the same circumstances, the difference in the results using the two methods should be due mainly to the refractory period. Thus, the refractory period of the axon in figs 1,2 was $1.7 \mathrm{~ms}(14 \cdot 0-12 \cdot 3)$ at the $10 \%$ level, $0.7 \mathrm{~ms}(13.0-12.3)$ at the $25 \%$ level, $0.5 \mathrm{~ms}(12 \cdot 8-12.3)$ at the $50 \%$ level and $0.5 \mathrm{~ms}$ $(12 \cdot 6-12 \cdot 1)$ at the $100 \%$ level.

\section{Refractory period and strength of testing stimulus}

It was possible to study the refractory period of 25 motor units in five subjects when the strength of the testing stimulus, at the fibular head, was $10 \%, 25 \%$ and $50 \%$ above threshold. The conduction velocity of these motor units ranged from 30 to $41 \mathrm{~m} / \mathrm{s}$.

Fig 3 shows the mean values of the axonal refractory periods of these 25 motor units at each stimulus level. Different numbers of motor units were studied in each subject and the standard deviations in the figure are based upon the mean values from each of the subjects. The length of the axonal refractory period, when the stimulus strength was $10 \%$ above threshold, was $1.72 \pm$

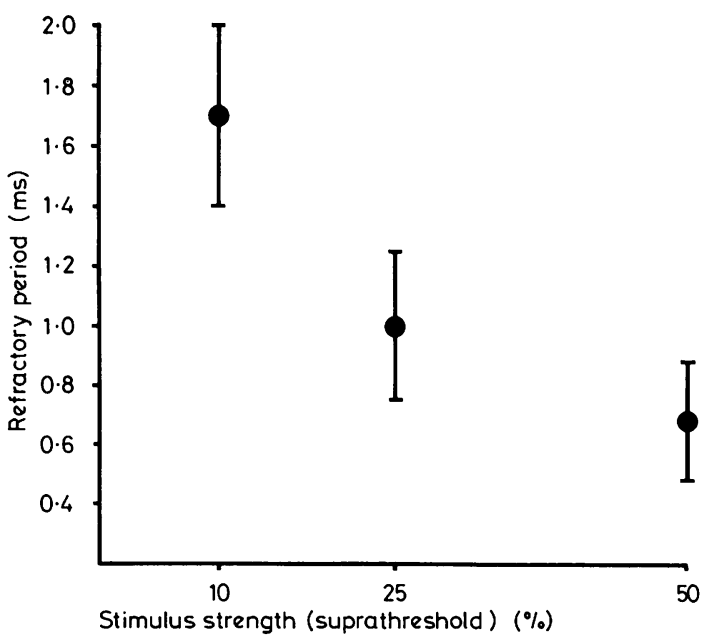

Fig 3 Refractory period and stimulus strength. Mean values of 25 motor units in five subjects. One standard deviation of the five individual mean values is marked.
$0.29 \mathrm{~ms}$. When the stimulus strength was $25 \%$ above threshold the refractory period was 0.99 . $0.26 \mathrm{~ms}$ and when stimulus strength was $50 \%$ above threshold the refractory period was $0.64 \pm$ $0.22 \mathrm{~ms}$. The difference of the refractory period between the $10 \%$ and the $25 \%$ above threshold stimulus levels was a mean of $0.73 \mathrm{~ms}(\mathrm{p}<0.01)$. The difference of the refractory period between the $10 \%$ and $50 \%$ levels was a mean of $1.08 \mathrm{~ms}$ $(\mathrm{p}<0.001)$.

It was not possible to check if there was any significant stimulus spread when the stimulus strength at the test point was increased from $10 \%$ to $50 \%$ above threshold; often it was necessary to reduce the number of stimulations as much as possible because of pain and displacement of the needle electrode. However, in 10 motor units the significance of the stimulus spread was investigated. No change of the latency difference between the distal and the proximal stimulus points was detected when stimulus strength at the fibular was increased from $10 \%$ to $25 \%$ above threshold. With an increase to $50 \%$ above threshold a shortening of $\mathbf{0 . 2} \mathrm{ms}$ was seen in two of the ten motor units, which means that the mean value of the refractory period at the $50 \%$ level noted above might be a little too low but nevertheless significantly lower than the mean value at the $10 \%$ level. For 19 of the 25 motor units the stimulus strength at the testing point could be further increased to $100 \%$ above the axonal threshold. The refractory period was then $0.52 \pm$ $0 \cdot 18 \mathrm{~ms}$. However, when this stimulus strength was used, the latency difference between the stimulus points exhibited a shortening of 0.2 or even $0.4 \mathrm{~ms}$ in six of the ten motor units.

\section{Refractory period in relation to conduction velocity}

Figs 1 and 2 show the findings from a motor unit with low axonal conduction velocity of $32 \mathrm{~m} / \mathrm{s}$. This motor unit had an axonal refractory period of $1.7 \mathrm{~ms}$ at $10 \%$ above threshold blocking stimulus.

Fig 4 shows the corresponding findings in a study of a motor unit with higher axonal conduction velocity. Fig 4A shows the only high amplitude potential on maximum voluntary effort. Fig 4B shows the results of electrical stimulation at the ankle and $C$ at the fibular head. The latency difference was $\mathbf{9 \cdot 2}$ ms corresponding to an axonal conduction velocity of 44 $\mathrm{m} / \mathrm{s}$. In D-F are shown the recordings during the blocking experiment with stimulus strength $10 \%$ above threshold. Interstimulus time was decreased from $8.2 \mathrm{~ms}$ (D) to $8.0 \mathrm{~ms}(\mathrm{E})$ and $7.8 \mathrm{~ms}(\mathrm{~F})$. 

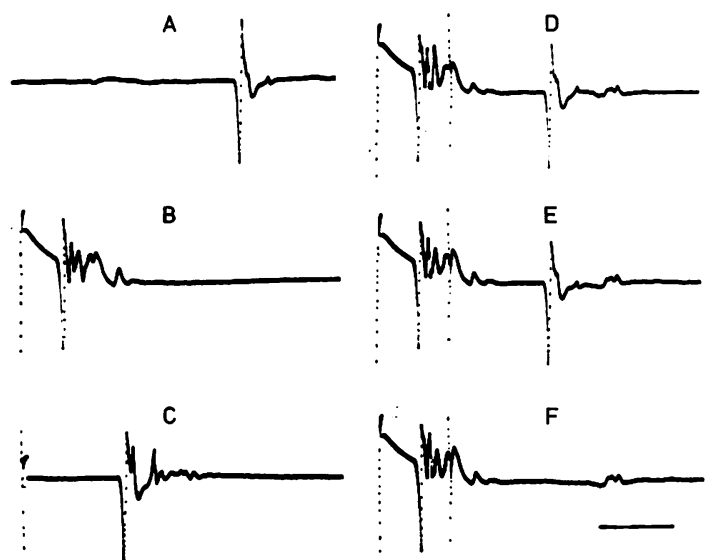

Fig 4 Axonal conduction velocity and refractory period. A: test unit on maximum voluntary effort. $B$ : test unit at stimulation at the ankle. C: test unit at stimulation at the fibula head. $D-F$ : test unit at the blocking experiment with decreasing interstimulus time. Time bar $10 \mathrm{~ms}$.

The shortest time interval without blocking was $8.0 \mathrm{~ms}$ and thus the refractory period at this stimulus level was $1.2 \mathrm{~ms}(9,2-8,0)$.

Fig 5A shows the relation between the axonal conduction velocity and refractory period studied with the stimulus strength at the fibular head at $10 \%$ above threshold. The total number of motor units were 71 in 12 subjects. The conduction velocity of these motor units ranged from 25 to $48 \mathrm{~m} / \mathrm{s}$. Of 15 motor units with an axonal conduction velocity below $35 \mathrm{~m} / \mathrm{s}$, nine had refractory periods longer than $1.8 \mathrm{~ms}$ and only one had a refractory period below $1.3 \mathrm{~ms}$. Of nine motor units with an axonal conduction velocity of 45 $\mathrm{m} / \mathrm{s}$ or more, five had refractory periods at or below $1.3 \mathrm{~ms}$ and none had a refractory period of $1.8 \mathrm{~ms}$ or longer.

Fig 5B shows the mean values of the axonal conduction velocities and refractory periods in each one of the 12 subjects. These data in the figure were correlated with a statistical significance at the $1 \%$ level $(p<0.01)$, the correlation coefficient being $-0 \cdot 71$. Fig $5 \mathrm{C}$ shows the axonal conduction velocity and refractory period of 23 motor units studied in one subject, JB. These individual data in the figure were correlated with a statistical significance at the $1 \%$ level $(p<0.01)$. The correlation coefficient was -0.55 .

\section{Refractory period and age}

The results presented in fig $5 \mathrm{~A}$ and $\mathrm{B}$ are based on studies of 45 motor units in elderly subjects and 26 motor units in young subjects (23 in the
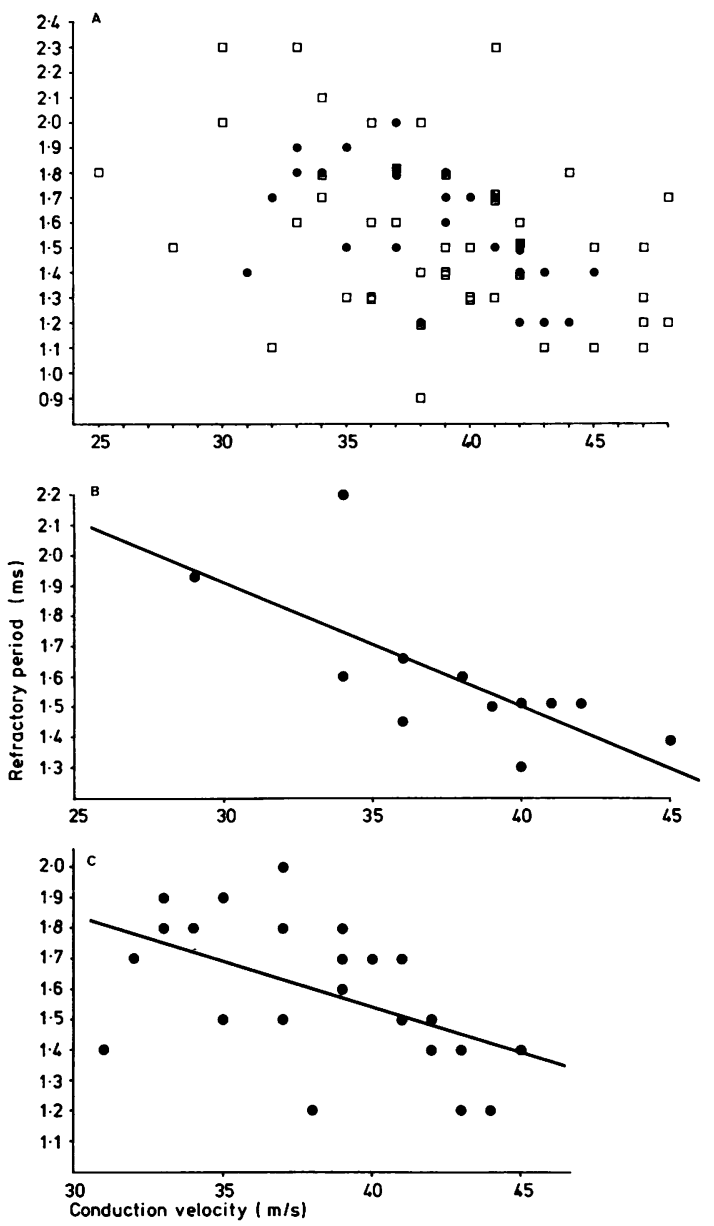

Fig 5 Axonal conduction velocities and refractory periods for 71 motor units. Forty-five motor units from nine elderly subjects (squares), 26 motor units from three young subjects (dots). B: Mean values of the conduction velocities and refractory periods of each of the 12 subjects. Correlation coefficient -0.71 . C: Axonal conduction velocities and refractory periods of 23 motor units in one subject. Correlation coefficient $-0 \cdot 55$. In $B$ and $C$ the regression lines are marked as illustrations.

author and three in two medical students). Regression analysis showed no significant difference between the age groups, suggesting that the same relationship between the conduction velocity and refractory period exists in both age groups.

The refractory period in relation to temperature If the room temperature was held below $20^{\circ} \mathrm{C}$ and no arrangements were made to keep the lower leg warm it was noted that during the examination the temperature at the fibular head 
often fell helow $30^{\circ} \mathrm{C}$, and that simultaneously the refractory period became longer even though the conduction velocity remained the same.

The effect of cooling the region around the fibular head to a skin temperature of $20^{\circ} \mathrm{C}$ was studied in two motor units in JB. The refractory period with the stimulus strength $10 \%$ above threshold at the fibular head was prolonged from 1.8 to $4.6 \mathrm{~ms}$ in one unit and from 1.8 to $3.0 \mathrm{~ms}$ in the other. After spontaneous rewarming to $28^{\circ} \mathrm{C}$ the refractory period was $2.8 \mathrm{~ms}$ and 2.2 $\mathrm{ms}$, that is, was prolonged by 1.0 and $0.4 \mathrm{~ms}$ respectively. The refractory period became normal when skin temperature increased to $32^{\circ} \mathrm{C}$. After warming to a skin temperature of $38^{\circ} \mathrm{C}$ a slight shortening of the refractory period took place. The conduction velocity was not significantly changed perhaps because only a short length of the actual nerve segment was superficial enough to be affected by the skin temperature variation.

\section{Discussion}

A systematic study of the refractory period of single human nerve fibres has not been made before. The actual method for measuring the refractory period does not, of course, achieve the exactness of animal experiments. The refractory period measurements were made after a propagated antidromic nerve impulse, in contrast to those measurements made after a conditioning electrical nerve stimulation. Moreover, it must be pointed out that muscle preparations resulting in a reduced number of motor units and an increased fibre density were necessary prerequisites and secondary changes in the proximal nerve segment, where measurements were made, cannot be excluded. Finally, stimulus spread might be a source of error in determining refractory period, even though it was shown to play a minor role in determining conduction velocity.

On increasing the test stimulus strength above the axonal threshold there was a decrease of the refractory period and an increase of the stimulus spread. Both these effects must be taken into account when the blocking technique is used with percutaneous recordings from whole muscles. If the stimulus strength at the testing point is $10 \%$ above the maximum percutaneous muscle response, those fibres having the highest threshold are stimulated $10 \%$ above threshold. According to the present findings the correction for the refractory period of these fibres should be about $1.7 \mathrm{~ms}$. Since there is often a threshold difference of more than $100 \%$ between different motor units this means that the motor units with the lowest thresholds are stimulated more than $100 \%$ above threshold when the nerve trunk is stimulated $10 \%$ above the maximum muscle response. The correction for the refractory period for these fibres should be about $0.6 \mathrm{~ms}$.

Stimulus strength at $10 \%$ above the percutaneously recorded maximum muscle response is thus accompanied by an unsafety factor of more than $1 \mathrm{~ms}$ when the correction for the refractory period is made. This means a possible error of more than $10 \%$ in calculating the conduction velocity of the peroneal nerve and even higher where shorter nerve segments are studied, for example the ulnar nerve. Much of this error can be avoided if the stimulus strength is high. In previous reports the stimulus strength often was not defined, but usually was $10 \%$ above maximal probably due to discomfort at higher stimulus strength. According to the present study the stimulus strength should be $50 \%$ above the percutaneously recorded maximal muscle response and the correction for the refractory period then $-0.6 \mathrm{~ms}$.

An inverse relationship between the axonal conduction velocity and the relative refractory period was observed when the strength of the test stimulus at the fibular head was $10 \%$ above threshold. Proper temperature control excludes the possibility that this was secondary to influence of temperature. This kind of relationship is compatible with findings in animal experiments. ${ }^{1924}$ The difference between the refractory period of an axon with a conduction velocity of $45 \mathrm{~m} / \mathrm{s}$ and of an axon with a conduction velocity of $30 \mathrm{~m} / \mathrm{s}$ is about $0.5 \mathrm{~ms}$ based upon the regression analysis in fig 5B. Thus, if the mean value of the refractory period at the $10 \%$ level is used when calculating the conduction velocity of both the slow and fast fibres an error of about $2 \mathrm{~m} / \mathrm{s}$ is introduced. Theoretically the fastest conducting fibres have the lowest threshold, which means that the error related to different stimulus strength is accentuated by the error related to different conduction velocities.

If there is the same relationship between the absolute refractory period of slow and fast conducting fibres and their relative refractory period, the absolute difference between the refractory period for the fast and slow fibres should be less at high stimulus level. When test stimulus strength was $50 \%$ above threshold, no statistically significant difference between the refractory period of fibres with different conduction velocities was observed. However, only a small number of motor units within only one segment of the con- 
duction velocity spectrum were studied at this stimulus level. Thus, no recommendations can be made concerning different corrections for the refractory period of the different fibres. However, the error introduced by using $0.6 \mathrm{~ms}$ for both slow and fast fibres is unlikely to be important.

The influence of temperature on the refractory period, well known from animal experiments ${ }^{25} 26$ is of great significance when using the blocking technique. With only a slight decrease of the skin temperature the refractory period is prolonged even when the conduction velocity is not significantly affected. This is because the nerve segment in which the refractory period is measured is more superficial, and thus more affected by external temperature variations than the rest of the nerve.

The relationship between the refractory period and the conduction velocity was the same in the young and the old age groups. This means that no change of the refractory period, other than that correlating to changes in conduction velocity, was observed in elderly persons when this method was used. It must be emphasised, however, that the elderly subjects were selected from a pensioners' gymnastic group and probably represent the most healthy persons in their age group. Moreover, the actual refractory period measurements were made at the fibular head and tell us nothing about the situation more distally. Furthermore, the young age group is mainly represented by one person.

To summarise, it seems possible to improve the reliability of the blocking technique in clinical practice by using a high stimulus level, which unfortunately often is painful, and by perfect temperature control. Of course factors other than refractory period are of great importance when optimising the blocking technique described by Hopf, but these aspects were beyond the scope of the study. Finally, since a proper correction for the refractory period is of such importance for the reliability of the method, knoweldge of the refractory period in pathological states is necessary if this method is to be used in the clinic.

\section{References}

1 Hopf HC. Untersuchungen über die Unterschiede in der Leitgeschwindigkeit motorischer Nervenfasern beim Menschen. Dtsch $Z$ Nervenheilk 1962; 183:579-88.

2 von Helmholtz $\mathbf{H}$. Messungen über den zeitlichen Verlauf der Zuckung animalischer Muskeln und die Fortpflantungsgeschwindigkeit der Reizung in den Nerven. Jona Müllers, Arach Anat Physiol 1850; 276-364.

3 Hodes R, Larrabee MC, German W. The human electromyogram in response to nerve stimulation and the conduction velocity of motor axons. Arach Neurol Psychiat 1948; 60:340-95.

4 Thomas PK, Sears TA, Gilliatt RW. The range of conduction velocity in normal motor nerve fibres to the small muscles of the hand and foot. J Neurol Neurosurg Psychiatry 1959; 22:175-81.

5 Hopf HC. Electromyographic study on so-called mononeuritis. Archives Neurol 1968; 9:307-12.

6 Miglietta $O$. Nerve motor fibre characteristics iı chronic ischemia. Arch Neurol 1966; 14:443-53.

7 Miglietta $O$. Motor nerve fibres in amyotrophic lateral sclerosis. Am J Phys Med 1968; 47: No 3, 118-24.

8 Chaco J. Conduction velocity of motor nerve fibres in progressive spinal atrophy. Acta Neurol (Scand) 1970; 46:119-22.

9 Seppäläinen A-M, Hernberg S. Sensitive technique for detecting subclinical lead neuropathy. Br J Ind Med 1972; 29:443-9.

10 Seppäläinen A-M. Peripheral neuropathy in forest workers. A field study. Work Environm Health 1972; 9:106-11.

11 Blackstock E, Rushworth G, Gath D. Electrophysiological studies in alcoholism. J Neurol Neurosurg Psychiatry 1972; 35:326-34.

12 Betts RP, Johnston DM, Brown BH. Nerve fibre velocity and refractory period disturbances in nerve trunks. J Neurol Neurosurg Psychiatry 1976; 39:694-700.

13 Gilliatt RW, Willison RG. The refractory and supernormal periods of the human median nerve. J Neurol Neurosurg Psychiatry 1963; 26:136-47.

14 Lowitzsch K, Hopf HC. Refraktärperioden und frequente Impulsfortleitung im gemischten $n$. ulnaris des Menschen bei Polyneuropathien. $Z$ Neurol 1973; 205:123-44.

15 Tackman W, Lehmann HJ. Refractory period in human sensory nerve fibres. Europ Neurol 1974; 12:277-92.

16 Kimura JA. Method for estimating the refractory period of motor fibres in human peripheral nerve. J Neurol Sci 1976; 28:485-90.

17 Kimura J, Yamada T, Rodnitzky L. Refractory period of human motor nerve fibres. J Neurol Neurosurg Psychiatry 1978; 41:784-90.

18 Kopec J, Delbeke, McComas AJ. Refractory period studies in a human neuromuscular preparation. J Neurol Neurosurg Psychiatry 1978; 41:54-64.

19 Paintal AS. Conduction in mammalian nerve fibres. In: Desmedt, ed. New Developments in Electromyography and Clinical Neurophysiology. vol 2. Basel: Karger, 1973; 19-41.

20 Bergmans J. The physiology of single human nerve fibres. Louvain: Vander, 1970.

21 Borg J, Grimby L, Hannerz J. Axonal conduction velocity and voluntary discharge properties of individual short toe extensor motor units in man. 
J Physiol (Lond) 1978; 277:143-52.

22 Jennekens FGI, Tomlinson BE, Walton JN. Data on the distribution of fibre types in five human limb muscles. J Neurol Sci 1971; 14:245-57.

23 Lambert EH. The accessory deep peroneal nerve. A common variation of extensor digitorum brevis. Neurology (Minneap) 1969; 19:1169-76.

24 Paintal S. Effects of temperature on conduction in single vagal and saphenous myelinated nerve fibres of the cat. J Physiol (Lond) 1965; 180: 20-49.

25 Adrian ED. The recovery process of excitable tissues, Part 2. J Physiol (Lond) 1921; 55:193-225.

26 Tasaki I. The excitatory and recovery process in the nerve fibre as modified by temperature changes. Biochim Biophys Acta 1949; 3:498-508. 